\title{
Bioinsecticida de capsaicinoides para el control de acanthoscelides obtectus (Say) (gorgojo) de frejol rojo (Phaseolus vulgaris L.) almacenado
}

\section{Capsaicinoid bioinsecticide for the control of acanthoscelides obtectus (Say) (weevil) in stored red bean (Phaseolus vulgaris L.)}

\author{
'Gamarra Mendoza, N.N.; ' ${ }^{2}$ Gamarra, P. R.; ${ }^{3}$ Tito, L. R.; ${ }^{4}$ Córdova, S.
}

Facultad de Ingeniería en Industrias Alimentarias / Universidad Nacional del Centro del Perú

Email:nngamarra@uncp.edu.pe

\section{Resumen}

Generalmente, los granos de frejol seco almacenados, son atacados por gorgojos, que causan un impacto negativo desde el punto de vista socioeconómico a pequeño y medianos productores de estas leguminosas; para contrarrestar a estos insectos plaga, utilizan insecticidas sintéticos altamente peligroso para la salud y contaminación ambiental.

Por ello, se evaluó el efecto de extractos de capsaicinoides de placenta (residuo) de ají panca en el control de acanthoscelides obtectus (Say) en frejol almacenado.

Se realizó la extracción de los capsaicinoides en medio etanólico a $60{ }^{\circ} \mathrm{C}$ con una velocidad de agitación de 150 rpm por $4 \mathrm{~h}$. Se cuantificó la concentración de capsaicinoides por HPLC. Los extractos fueron evaporados y resuspendidos en metanol al $4 \%$ y $2 \%$ de diclorometano. A partir del extracto, se preparó diluciones de $0 \%$ (To), $10 \%$ (T1), $25 \%$ (T2), $50 \%$ (T3) y $100 \%$ (T4), los cuales fueron expuestos con 10 unidades de gorgojos por cada tratamiento en 30 y $60 \mathrm{~min}$. La mortandad de los gorgojos alcanzó de 20 a 35\% con los tratamientos T1 a T4 a los $30 \mathrm{~min}$. Sin embargo, a los $60 \mathrm{~min}$. de exposición la mortalidad fue $\geq$ al $90 \%$ en todos los tratamientos excepto en To. Los capsaicinoides tienen propiedades potenciales biócidas para el control de estos insectos plaga en almacén compatible con el medio ambiente.

Palabras clave: acanthoscelides obtectus, capsaicinoides, insecto plaga, biócida, leguminosa.

\begin{abstract}
Generally, stored dry bean grains, are attacked by weevils, which cause a negative impact to small and medium producers of these legumes from a socioeconomic point of view; synthetic insecticides highly dangerous for health and environmental pollution are used to remove pest insects.

In this regard, the effect of extracts of placental capsaicinoids (residue) of ají panca in the control of Acanthoscelides obtectus (Say) in stored beans was evaluated.

Capsaicinoids were extracted in ethanolic medium at $60{ }^{\circ} \mathrm{C}$ with a stirring speed of $150 \mathrm{rpm}$ for $4 \mathrm{~h}$. Capsaicinoid concentration was quantified by HPLC. The extracts were evaporated and resuspended in 4 $\%$ methanol and $2 \%$ dichloromethane. Dilutions of $0 \%$ (To), $10 \%$ (T1), $25 \%$ (T2), $50 \%$ (T3) and 100 $\%$ (T4) were prepared from the extract, which were exposed with 10 units of weevils for each treatment in 30 and $60 \mathrm{~min}$. The mortality of the weevils reached 20 to $35 \%$ with treatments T 1 to T4 at $30 \mathrm{~min}$. However, at 60 minutes exposure, mortality was $\geq 90 \%$ in all treatments except I To. Capsaicinoids have potential bioside properties for controling these insects in storage that are compatible with the environment.
\end{abstract}

Keywords: acanthoscelides obtectus, capsaicinoids, pest insect, biocide, legume 


\section{Introducción}

Las leguminosas de grano, conforman un importante grupo de alimentos, que han desempeñado un papel fundamental en la alimentación de casi todas las civilizaciones del mundo desde hace más de 20 mil años. En América, el frijol y el pallar, constituían alimentos muy apreciados en las culturas prehispánicas, como la Inca, Maya y Azteca, desde hace más de 5000 años (Valladolid, 2016). Nuestros antepasados incas legaron a la humanidad los frijoles y pallares más vistosos y grandes del mundo y frijoles que tienen la capacidad de expandirse, similar al maíz pop corn, cuando es sometido a un proceso de tostado. (Valladolid, 2016).

Con el descubrimiento de América, el frijol se difundió por toda Europa y el mundo, que fue considerado como una nueva fuente alimenticia muy nutritiva. Desde entonces, el frijol común se ha convertido en la leguminosa de grano más importante del mundo (Cubero y Moreno, 1983). La India, China, Canadá y Brasil, son los más importantes productores de legumbres secas. El volumen de producción de estos granos, se ubican en el cuarto lugar de importancia después del trigo, maíz y arroz (FAOSTAT; 2011, 2012). En Latinoamérica se cultivan 15 especies de leguminosas y en el Perú 13 de las cuales 10 ocupan áreas de siembra significativas. En el Perú se cultiva más del $86 \%$ del total de las especies, lo que sugiere fomentar su producción. Los frijoles son una de las principales fuentes de proteínas, con un alto contenido de hierro, elemento vital para el buen desarrollo cerebral, cada 100 gramos, contiene $20 \mathrm{~g}$ de proteínas, 5.8 g de grasa y más de $3 \mathrm{~g}$ de fibra (Ulloa et al., 2011).

Sin embargo, uno de los problemas que enfrenta el agricultor es el daño causado por los agentes biológicos durante el almacenamiento de frijol, las plagas que atacan en su almacenamiento tienen gran importancia económica para cualquier país. De estas, los insectos son el principal causante, ocasionando pérdidas cuantiosas debido a que deterioran directa e indirectamente a los granos haciéndole orificios o graderías, volviéndolo inaceptable para consumo humano. La especie que causa mayor daño al grano de frijol almacenado es Acanthoscelides obtectus, comúnmente denominado "gorgojo del frijol" (Gutiérrez, 2016) o escarabajo de semillas (Ramírez, 2015), perteneciente a la orden coleóptera y a la familia bruchidae. Se originó en Suramérica y están ampliamente distribuidas desde Chile hasta los Estados Unidos (Schoonhoven et al., 1988). En sus estadios inmaduros se alimentan de las semillas de alrededor de 34 familias de plantas, principalmente de leguminosas y tiene gran impacto en las áreas agroalimentarias (De La Cruz et al., 2013). Por su frecuencia y daños, es señalado como la plaga más importante del frijol a escala mundial (D“Aubeterre et al., 2012). Esta especie infesta los granos en el campo y continúa durante el almacenamiento (Dell'Orto 1985). En el campo ocasiona pérdidas hasta del $20 \%$, mientras que en granos almacenados puede afectar hasta el $100 \%$ (Dell'Orto 1985).
El daño económico que ocasiona el A. obtectus es importante porque los granos afectados pierden parcial o totalmente su valor comercial, se produce pérdidas del peso, disminución de la capacidad germinativa y disminución de su valor nutritivo (3). Los insectos dejan restos de heces e individuos que mueren durante su desarrollo en los granos; adicionalmente son infestados por hongos y otros patógenos que disminuyen también la calidad del grano, por lo que es rechazado para el consumo humano.

Estos mismos insectos plaga, se encontraron en garbanzos, lentejas y arvejas. Sin embargo, existen escasos trabajos sobre la biología de estas especies, debido a que son ágiles caminadores y voladores, el desarrollo de la larva, prepupa y pupa ocurre dentro del grano, así como a su capacidad de fingir estar muertos cuando son perturbados; aspectos estos que dificultan notablemente su estudio (Ramírez y Suri, 2015). El daño que causa estos insectos plaga a los frijoles, obliga a los agricultores a vender la cosecha rápidamente, incluso en épocas en que la oferta es alta y los precios son muy bajos en el mercado, estas afectaciones suponen un destrío automático de la producción al observar más del $10 \%$ de semillas con el insecto u orificio de salida (Bermejo, 2011).

Frente a estos hechos los pequeños agricultores aplican tratamientos convencionales como es el fosfuro de aluminio o fosfuro de magnesio, que actúa como fumigante. Sin embargo, su uso está prohibido por su toxicidad y peligrosidad, a ello se suma la mala manipulación de los pequeños agricultores, poniendo en riesgo tanto su salud como la sanidad ambiental (Martínez, 2008). Estos hechos sugieren la necesidad de encontrar tratamientos alternativos contra estas plagas de almacén, así como el uso de bioinsecticidas o metabolitos secundarios, como los capsaicinoides de la placenta (residuo) de ají panca que tienen efecto repelente y biosida de insectos (Claros, 2018) y de algunos otros depredadores en determinadas dosis de aplicación.

Estos hechos sugieren la necesidad de llevar a cabo una gestión de control adecuado evitando posibles impactos ecológicos y económicos negativos en los próximos años. El uso de vegetales o residuos agroindustriales con propiedades insecticidas es una de las principales alternativas ecológicamente amigables y económicamente viables. Existen tendencias recientes de aumentar la producción y uso de bioinsecticidas (Gupta \& Dikshit, 2010; Mazid et al., 2011). En el presente trabajo de investigación se experimentó el efecto biotóxico de capsaicinoides sobre los Acanthoscelides obtectus o gorgojo de frijol en poscosecha.

\section{Materiales y métodos}

\section{Preparación del extracto de capsaicinoides}

Los ensayos experimentales se realizaron en el Laboratorio de Química de Alimentos de la Facultad de Ingeniería de Industrias Alimentarias. La colecta de placentas (residuo) de ají panca se realizó de la empresa Sasón Lopesa de Huancayo 
y fue trasladado al laboratorio de investigación. La placenta libre de semillas se deshidrató en una secadora de cabina a $50{ }^{\circ} \mathrm{C}$ hasta una humedad de 8 a $10 \%$. Luego fue molido y tamizado hasta un tamaño de partícula de 0,25 a $0,42 \mathrm{~mm}$, del cual se realizó la extracción de capsaicinoides con etanol al $99 \%$ (marca EMPARTA $\AA$, adquirido de Merck), a $60{ }^{\circ} \mathrm{C}$, agitado en $270 \mathrm{rpm}$ por $12 \mathrm{~h}$ (Gavilán, 2018), los extractos fueron analizados por cromatografía líquida de alta eficiencia (HPLC), se utilizó un cromatógrafo marca Shimadzu, adquirido de Electromédica Peruana SAC, previamente se determinó los cromatogramas en diferentes concentraciones de los patrones de capasaicinoides: nordihidrocapsaicina, capsaicina y dihidrocapsaicina, con los cuales se identificó y cuantificó los capsaicinoides de los extractos de placenta de ají panca; para ello, se elaboró una curva de calibración. Los extractos de mayor contenido de capsaicinoides totales fueron almacenados a $4{ }^{\circ} \mathrm{C}$ hasta el momento de su aplicación en los gorgojos de frijol.

Bioensayos toxicológicos con extractos de capsaicinoides en A. obtectus de frijol rojo

La colección de los A. obtectus, se realizó de los almacenes de maíz (figura 1) en el distrito de Chilca, provincia de Huancayo. Se utilizó el método de Gutiérrez (2016) y Da Silva (2017), con algunas modificaciones. Se colectó los gorgojos de frijoles almacenados en un recipiente de polietileno de alta densidad y se trasladaron al laboratorio, seguidamente cada 10 unidades de gorgojos adultos fueron colocados en cada placa Petry de 100 x 15 mm y luego se preparó diluciones de extractos de capsaicinoides en concentraciones de $0 \%$ (To); $10 \%$ (T1); $25 \%$ (T2), 50 $\%$ (T3) y $100 \%$ (T4) (v/v), haciendo cinco tratamientos (To - T4) que fueron aplicados directamente por aspersión sobre los insectos y se mantuvo en contacto por $30 \mathrm{y}$ 60 min (bloques), luego se evaluó la mortandad contando los insectos muertos después de cada tiempo de contacto.

a)

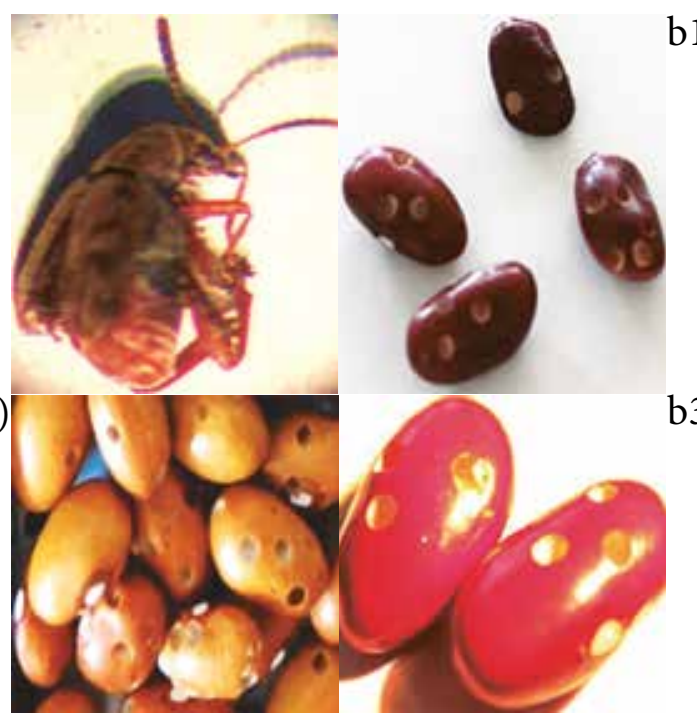

Figura 1

a) Acanthoscelides obtectus; b1), b2) y

b3) Granos de frejol con orificios dañados por A. obtectus
Se utilizó un diseño estadístico bloque completamente aleatorio (DBCA) para organizar y llevar a cabo el diseño experimental. Los datos que resultaron del diseño experimental se analizaron mediante un análisis de varianza con un nivel de error al $5 \%$ y luego se utilizó una prueba de medias de Tukey para determinar la diferencia significativa entre tratamientos y bloques. Los datos obtenidos fueron procesados mediante el software Statistical Analitical Software (SAS) V 9.1. y los gráficos con el programa Excel.

\section{Resultados y discusión}

Cuantificación de capsaicinoides de los extractos de placenta de ají panca

La cantidad de capsaicinoides de los extractos de placenta (no comestible) fueron 5,7 veces mayor que el pericarpio (parte comestible) del fruto de ají panca, siendo la capsaicina (CAP) la que predomina, seguido por la DHC y en menor cantidad la NDHC (Figura 2) (Gavilán et al., 2018). La cantidad de capsaicinoides totales fue de $1,96 \mathrm{mg} / \mathrm{ml}$ del extracto. Los capsaicinoides se biosintetizan principalmente en la placenta de los frutos de ají (Nowaczyk et al., 2006a y 2006b; Pandhair \& Sharma, 2008), y desde esta parte anatómica se distribuye o difunde a las otras estructuras del fruto y de la planta (Buczkowska et al., 2016).

Se prepararon las diferentes diluciones de capsaicinoides del extracto a partir de la concentración total del extracto de ají panca y se aplicó a los insectos plaga.

Figura 2

Cromatograma de capsaicinoides de placemta de ají panca: $\mathrm{NDHC}=$ Nodihidrocapsaicina, $\mathrm{CAP}=$ capsaicina, DHC $=$ Dihidrocapsicina .

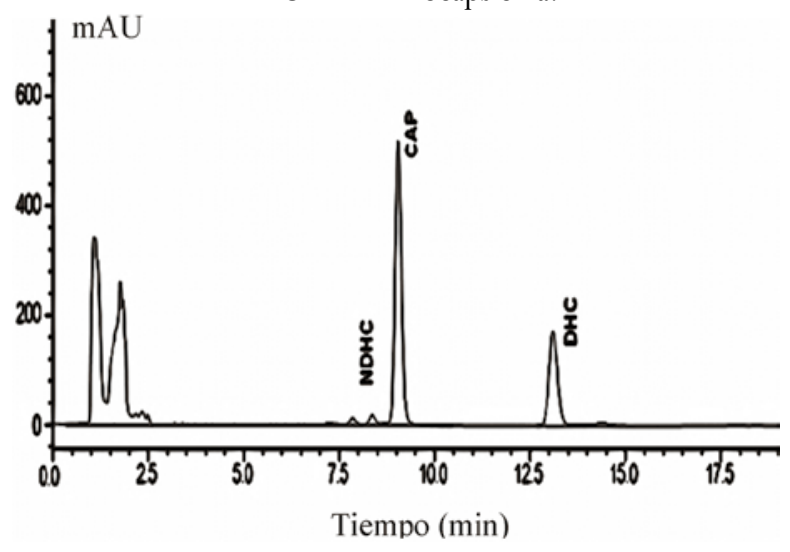

Porcentaje de mortalidad del A. obtectus en diferentes concentraciones de capsaicinoides

En la Tabla 1, se observa los porcentajes de mortalidad del gorgojo de frijol en relación a las diferentes diluciones y tiempos de exposición con el bioinsecticida de capsaicinoides, probados en los bioensayos toxicológicos. 
Tabla 1

Porcentaje de mortalidad de A. obtectus con capsaicinoides

\begin{tabular}{ccc}
\hline \multicolumn{1}{c}{ Tratamiento } & \multicolumn{2}{c}{$\%$ de mortalidad } \\
(\% de capsaicinoides) & $\begin{array}{c}\text { Bloque I } \\
(30 \mathrm{~min})\end{array}$ & $\begin{array}{c}\text { Bloque II } \\
(60 \mathrm{~min})\end{array}$ \\
\hline To (control) & 0 & 0 \\
T1 (10\%) & $25( \pm 7.1)$ & $90( \pm 14)$ \\
T2 (25\%) & $35( \pm 7.1)$ & $80( \pm 0)$ \\
T3 (50\%) & $30( \pm 0)$ & $100( \pm 0)$ \\
T4 (100\%) & $20( \pm 14)$ & $100( \pm 0)$ \\
\hline
\end{tabular}

Nota: Promedio de tres repeticiones

A los 30 minutos de exposición de los gorgojos en los tratamientos T1, T2, T3 y T4, alcanzó de 20 a $35 \%$ de mortandad y; a los 60 minutos fue superior a $79 \%$ de mortalidad y en los tratamientos T3 y T4, reportó al $100 \%$. Se observa que conforme se incrementa el tiempo de contacto entre los gorgojos y los bioinseticidas la mortandad se incrementa en aproximadamente de 45 a $80 \%$ (Figura 3).

\section{Figura 3}

Mortalidad de S. zeamais en diferentes tiempos de exposición sobre concentraciones distintas de capsaicinoides $(\mathrm{To}=0 \% ; \mathrm{T} 1=10 \% ; \mathrm{T} 2=25 \%, \mathrm{~T} 3=50 \%) \mathrm{yT} 4=100 \%$

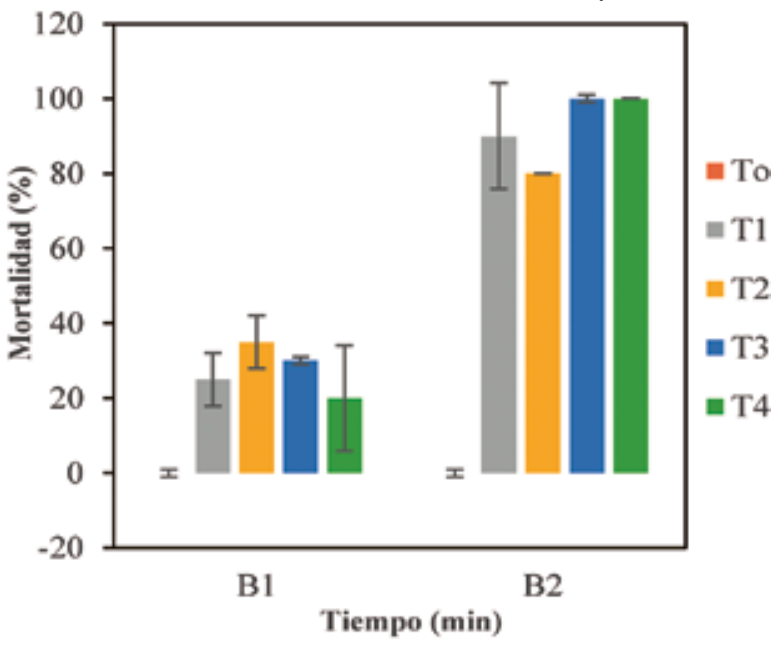

Según al análisis de varianza en la Tabla 2, se observa que existe efecto significativamente diferente $(\mathrm{P}<0.05)$ entre los tratamientos y los tiempos de exposición. El efecto de las diferentes concentraciones de capsaicinoides utilizadas en la biotoxicidad de los gorgojos superó la Dosis Letal Media (DL50) a los 60 minutos de exposición.

Tabla 2

Análisis de varianza de los tratamientos y bloques en la mortandad de A. obtectus

\begin{tabular}{lccccc}
\hline Fuente & $\begin{array}{c}\text { Grados de } \\
\text { libertad }\end{array}$ & $\begin{array}{c}\text { Suma de } \\
\text { cuadrados } \\
\text { del ANOVA }\end{array}$ & Cuadrado & & \\
medio & Valor & Pr $>$ F & Provern \\
\hline Tratamientos & 4 & 11670.0 & 2917.5 & 9.02 & $<.0008$ \\
Bloques & 1 & 13520.0 & 13520.0 & 41.78 & $<.0001$ \\
\hline
\end{tabular}

Relacionando las observaciones realizadas durante el tiempo que duro los bioensayos, podemos afirmar que las diferentes concentraciones de los bioinsecticidas de capsaicinoides tuvieron efectos favorables en el control de gorgojos de frijol, ya que el número de insectos muertos era superior comparado al control, en este último la población se mantuvo estable. Los signos de muerte de los gorgojos, mostraban inamovilidad, compresión o extensión de patas, extensión de alas posteriores en el extremo final del abdomen y descanso en posición dorsal o lateral, como se muestra en la Figura 4.

\section{Figura 4}

a) A. obtectus tratado con extractos de capsaicinoides, b) A. obtectus muertos

a)

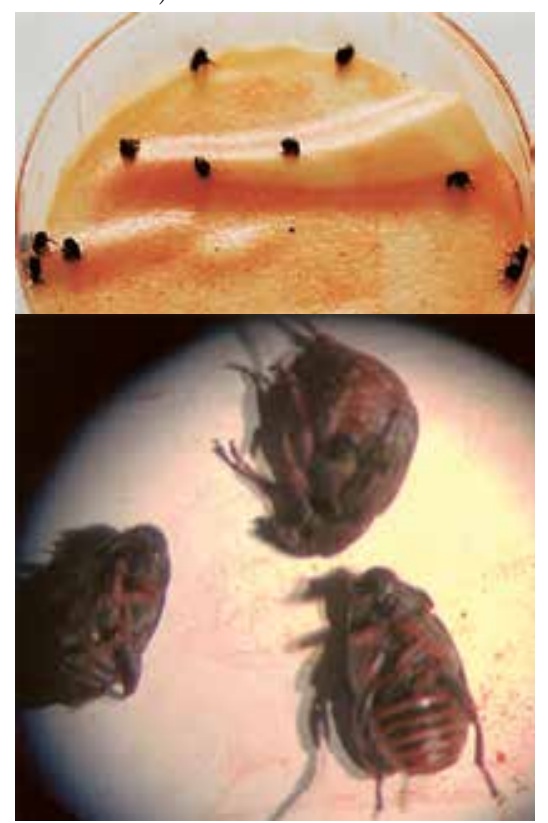

Da Silva (2016), realizó el control biológico de A. obtectus en judías, utilizando aceites esenciales de vegetales, con una dosis de $1 \mu \mathrm{l}$ de aceite de romero, logró mayor mortalidad (53,3\%) de A. obtectus, que aceite de geranio $(15,0 \%)$, canela $(10,0 \%)$ y verbena $(1,7 \%)$. Y con una dosis de $3 \mu \mathrm{l}$, obtuvo significativamente superior la muerte de A. obtectus adultos de 85,0 \% comparado, a las obtenidas con los otros aceites esenciales, que alcanzó de $25 \%$ a $40 \%$. Así mismo, el investigador destaca que el tiempo de exposición fue de tres horas de los A. obtectus en diferentes dosis de aceite esencial de romero, donde la mortalidad fue superior al 80,0\% en todas las dosis evaluadas.

Se sabe que los aceites esenciales de plantas poseen actividades insecticidas y/o repelentes frente a los insectos y, además, no representan amenaza para el medio ambiente o para la salud humana, convirtiéndose de esta forma en una alternativa posible a los insecticidas sintéticos (Jumbo et al., 2014). Las diferentes actividades de aceites esenciales en huevos y 
adultos han sido descritas para algunos insectos (Yang et al. 2003; Yang et al., 2005; Abd El-Salam 2010), y específicamente para A. obtectus. Papachristos y Stamopoulos (2002) y Regnault-Roger y Hamraoui (1994), han demostrado que la capacidad de las larvas para entrar en las alubias y las susceptibilidades larva/ pupa son afectadas diferencialmente por cada tipo de aceite esencial evaluado.

Por otro lado, Gutiérrez (2016), realizó un estudio para determinar el efecto de la Beauveria bassiana, sobre el biocontrol del gorgojo del frijol Acanthoscelides obtectus, utilizó una sola dosis de este bioinsecticida, fue puesto en contacto con los gorgojos por sumersión, aspersión y en polvo seco y se probó en condiciones ventiladas y no ventiladas, el tratamiento que mejor respuesta presentó, fue en los gorgojos que no eran ventilados, alcanzó una mortalidad de 86.43 \% y el que ejerció mayor efecto fue el polvo seco siendo de $100 \%$. Las esporas del hongo B. bassiana, al entrar en contacto con la cutícula del insecto, germina, se forma y produce micelio que crece encima de la cutícula y penetra en el interior del cuerpo del hospedero (Tecnologías Naturales Internacional, 2014).

Investigaciones sobre diferentes tipos de preparados de plantas, como polvos, extractos de solventes, aceites esenciales y plantas enteras, presentan actividad insecticida, incluida su acción como fumigantes, repelentes, antialimentarios, antioviposición y reguladores del crecimiento de insectos (Isman, 2000). Prabhakaran et al., (2017), señala que, el uso de sustancias de plantas nativas en forma de aceites, extractos de hojas, raíces, frutas y semillas se ha evaluado desde hace siglos; las plantas producen diversos compuestos bioactivos (metabolitos secundarios) como alcaloides, glucósidos, flavonoides, taninos y saponinas para su supervivencia contra plagas y enfermedades; así como, para atraer a los insectos para su polinización. Stark y Banks (2003), sostienen que las concentraciones de metabolitos secundarios de los diferentes extractos de plantas aplicados en poblaciones de insectos, estabilizan su crecimiento y seguidamente se extinguen; cabe destacar que dependiendo de la dosis de aplicación de los extractos de plantas pueden actuar como plaguicidas o repelentes, ya que a diluciones más altas, los efectos serán más satisfactorios, se corrobora con los bioensayos de García et al., (2014), donde la tasa instantánea de crecimiento poblacional de $T$. vaporariorum tuvo un crecimiento lineal negativo, a medida que se incrementó las concentraciones de los extractos de higuerilla, anon y eucalípto.

Otro factor que influye en el efecto insecticida de los extractos fitoquímicos, es la composición y naturaleza química del metabolito secundario, estos compuestos se biosintetizan y se distribuyen diferencialmente entre grupos taxonómicos, presentan diferentes propiedades bioactivas, muchos desempeñan funciones ecológicas y se caracterizan por sus diferentes usos y aplicaciones como medicamentos, insecticidas, herbicidas, fungicidas, bactericidas, aromatizantes o colorantes, entre otros. Reciben también la denominación de productos o compuestos naturales.

Comparando los resultados obtenidos en la presente investigación con los resultados de Castillo et al., (2012), quienes determinaron la actividad biológica in vitro del extracto de Capsicum chinense Jacq contra Bemisia tabaci Genn, demostraron que, en los bioensayos de mortalidad, las diluciones con 30 y $40 \%$ del extracto del fruto de C. chinense Jacq presentaron diferencias significativas con respecto al resto de las diluciones evaluadas. Así mismo, Claros et al., (2019), testearon el efecto de capsaicinoides y glucosinolatos de placenta de Capsicum chinense y de Tropaelum tuberosum en Aphis cytisorum de Spartium junceum L. la toxicidad de los compuestos se incrementó en relación a su concentración, causando un alto porcentaje de mortalidad de 83-99 \% cuando el Aphis era expuesto a diluciones de 10\% de capsaicinoides, 75-100\% de glucosinolates, o una mezcla de $10 \%$ capsaicinoides y $90 \%$ de glucosinolatos.

En estudios anteriores, realizados por Olszewska et al., (2010), demostraron claramente que la capsaicina incrementa la liberación de $\mathrm{CO} 2$ en larvas Tenebrio molitor en un promedio de 1.33 veces más alto que el grupo control y la aplicación simultanea de capsaicina y piretroides ocasionó la más alta liberación de $\mathrm{CO} 2$, lo que indica que hay un sinergismo entre capsaicina e insecticida, que genera un incremento de la tasa metabólica y por consiguiente la intoxicación del Tenebrio molitor. Edelson et al., (2002), mostraron que los capsaicinoides, aplicado como único componente biocida, causaba bajos porcentajes de mortalidad de $A$. persicae, pero actuando en mezcla con otros insecticidas genera efecto sinérgico que provoca altos niveles de mortalidad. Los diferentes estudios corroboran el efecto insecticida de los capsaicinoides, el presente estudio busca contribuir significativamente en el desarrollo de nuevos bioinsecticidas, recuperando los residuos de los frutos de ají de la industria de Capsicum y su aplicación en las áreas de almacenamiento y manejo poscosecha de granos, con posibilidades de ser masificado y producido a escala industrial. El presente estudio es un punto de partida inicial para confirmar las potencialidades de los capsaicinoides para su uso en el manejo de dichos insectos en almacenes de granos u otras instalaciones destinadas a la conservación de alimentos para animales. 


\section{Conclusiones}

- El efecto del bioinsecticida, resultó favorable en el control del gorgojo de frijol (A. obtectus), logrando porcentajes mayores al $79 \%$ de mortalidad en los bioensayos toxicológicos, después de los 60 minutos de contacto con las diferentes concentraciones de bioinsecticidas utilizadas. La utilización de los residuos de ají panca para la extracción de capsaicinoides y su aplicación como bioinsecticida, se enmarca en un contexto ecoamigable e inocuo a la salud humana y de animales.

\section{Referencias bibliográficas}

Abd El-Salam, A.M.E. (2010). Toxic and deterrent effects of two volatile oils against cowpea weevil, Callosobruchus chinensis (Coleoptera: Bruchidae). Archives of Phytopathology and Plant Protection 43 (16): 1596-1607.

Bermejo, J. 2011. Agrológica (en línea). ES, consultado 27 jun. 2014. Disponible en:http://www.agrologica.es/informacion-plaga/gorgojo-judia-acanthoscelides-obtectus /

Buczkowska, H. (2016). Relationships of the capsaicinoid content between the fruit parts of hot pepper (capsicum annuum 1.). Acta Scientiarum Polonorum-Hortorum Cultus. 15(4): 185-198.

Castillo-Sánchez, L. E.; Jiménez-Osornio, J. J.; Delgado-Herrera, M. A. (2012). Actividad biológica in vitro del extracto de Capsicum chinense Jacq contra Bemisia tabaci Genn. Revista Chapingo serie Horticultura. 18(3): 345-356.

Claros C. L. J.; Pinillos, O. E.;Tito, R.; Seguil M. C. and Gamarra, M. N. N. (2019). Insecticidal properties of capsaicinoids and glucosinolates extracted from Capsicum chinense and Tropaeolum tuberosum. Insect, 10, 132; doi: doi:10.3390/insects 10050132

Cubero, J.I. y M.T. Moreno (ed). (1983). Leguminosas de Grano. Ediciones Mundi Prensa. Madrid, España. 359 pp.

Da Silva, H. P. (2017). Control biológico del gorgojo de la judía Acanthoscelides obtectus (Say) (Coleoptera: Chrysomelidae: Bruchinae) en la región de Castilla y León -España. Tesis Doctoral. 1-161.

De La Cruz, A.; Romero, J.; Carrillo J. L.; García, E, Grether R, Sánchez S. (2013). Brúquidos (Coleoptera: Bruchidae) del Estado de Tabasco, México. Acta Zoológica Mexicana, 29:1-95.

D'Aubeterr R.; Principal, J.; Barrios, C.; Graterol, Z. (2012). Insectos plaga en vainas de Acacia tortuosa y Prosopis juliflora para consumo animal en las zonas semiáridas del Estado Lara, Venezuela. Zootecnia Trop. 30(2):147-153.

Dell' Orto H, Arias C. J. Insectos que dañan granos. Productos almacenados. Santiago de Chile. FAO, Serie: Tecnología Poscosecha 4. 1985; 46-49.

Edelson J. V.; Duthie J.; Roberts, W. (2002). Toxicity of biorational insecticides: activity against the green peach aphid, Myzus persicae (Sulzer). Pest Manag Sci. 58(3):255-60.

García, V. L. V.; Soto, G. A. y Bacca, T. (2014). Efecto insecticida de productos alternativos en Trialeurodes vaporariorum (Hemiptera: Aleyrodidae) Insecticide effect of alternative products on Trialeurodes vaporariorum (Hemiptera: Aleyrodidae). Revista Colombiana de Entomología 40 (2): 143-147.

Gavilán, G. N.; Tito, R.; Gamarra, M. N. (2018). Capsaicinoids and pungency in Capsicum chinense and capsicum baccatum fruits. Pesq. Agropec. Trop., Goiânia. 48(3): 237-244.

Gutiérrez, J. J. X. (2016). Manejo de Acanthoscelides obtectus (Say) en frijol común (Phaseolus vulgaris L.) almacenado utilizando Beauveria bassiana (Bals y Vui11). Trabajo de graduación. Facultad de Agronomía. Universidad Nacional Agraria. Managua (Nicaragua). $34 \mathrm{p}$

Gupta, S.; Dikshit, A. K. (2010). Biopesticides: An ecofriendly approach for pest control. Journal of Biopesticides. 3: 186-188.

Jumbo, LOV, LRA Faroni, y EE Oliveira. (2014). «Potential use of clove and cinnamon essential oils to control the bean weevil, Acanthoscelides obtectus Say, in small storage units». Industrial Crops and Products 56: 27-34.

Isman M.B. (2000). Plant essential oils for pest and disease management. Crop Protection, 19: 603-608.

Martínez M, Ibarra E. (2008). Pastilla del amor se vuelve delictiva. El Nuevo diario. 5 Abril 2008 p 11.

Mazid, S.; Kalita, J.C.; Rajkhowa, R. C. (2011). A review on the use of biopesticides in insect pest management. International Journal of Science and Advanced Technology. 1: 169-178.

Nowaczyk, P. et al. (2006a). Differences of capsaicinoids content in pericarp and paste of soft-flesh Capsicum spp. fruit. Folia Horticulturae. 18(2): 99-103.

Nowaczyk, P.; Nowaczyk, L.; Banach, M. (2006b). The capsaicin and dihydrocapsaicin contents in soft-flesh fruit of Capsicum frutescens L. and Cap- 
sicum annuum L. hybrids. Herba Polonica. 52(1): $38-42$.

Olszewska, J.; Têgowska, E.; Grajpel B.; and Adamkiewicz, B. (2010). Effect of application of capsaicin and pyrethroid on metabolic rate in mealworm Tenebrio molitor. Ecological chemistrya ndengine ering. 17(10): $129-133$.

Organización de las Naciones Unidas para la Agricultura y la Alimentación (FAOSTAT). (2011). Codex alimentarius-Cereales, legumbres, leguminosas y productos proteinicos vegetales. Primera Edición. Roma.

Organización de las Naciones Unidas para la Agricultura y la Alimentación (FAOSTAT). (2012). codex alimentarius-Cereales, Legumbres, Leguminosas y Productos proteinicos Vegetales. Primera Edición. Roma.

Pandhair, V.; Sharma, S. (2008). Accumulation of capsaicin in seed, pericarp and placenta of Capsicum annuum L. fruit. Journal of Plant Biochemistry and Biotechnology, 17(1): 23-27.

Papachristos, DP, y DC Stamopoulos. (2002). «Toxicity of vapours of three essential oils to the immature stages of Acanthoscelides obtectus (Say) (Coleoptera: Bruchidae)». Journal of stored products research 38 (4): 365-73.

Prabhakaran, P.; Radhakrishnan, B.; Srikumar, K. K.; and Suresh K. B. (2017). Efficacy of Certain Common Fernsagainst Red Spider Mite Oligonychus coffeae and Tea Mosquito Bug Helopeltis theivora Infesting Tea. Plant Protect. Sci. 53 (4), 232-242. doi: $10.17221 / 23 / 2015$-PPS.

Ramírez, S.; Suris, M. (2015). Ciclo de vida de Acanthoscelides obtectus (Say.) sobre frijol negro (Phaseolus vulgaris L.) en condiciones de laboratorio. Comunicación corta. Rev. Protección Veg. Vol. 30 No. 2 (mayo. -ago. 2015): 158-160 ISSN: 2224-4697.

Ramírez, M. M. (2015). Biología y hábitos de insectos de granos almacenados. Curso sobre insectos de granos y semillas de almacén. Aguas calientes. Ags. México, pag. $1-51$.

Regnault-Roger, C, y A Hamraoui. (1994). Inhibition of reproduction of Acanthoscelides obtectus Say (Coleoptera), a kidney bean (Phaseolus vulgaris) bruchid, by aromatic essential oils. Crop Protection 13 (8): 624-28.

Schoonhoven, A. V.; Cardona, C. y García, J. (1988). Principales insectos que atacan el grano almacenado y su control; guía de estudio para ser utilizada corno complemento de la unidad audiovisual sobre el mismo tema. 3 ed. Cali, Colombia. CIAT. 48 p.

Stark J.D., Banks J.E. (2003). Population level effects of pesticides and other toxicants on arthropods. Annual Review of Entomology, 48: 505-519.

Tecologías Naturales internacional. (2014). MicotrolMRES. SNT. (En linea). Consultado el 10 de febrero del 2015. Dispoible en http:// www.bactiva.com/index.php/ru/tecnologias/226-bioinsectisida.

Ulloa, J A, Rosas U, P, Ramírez R, J, C, Ulloa R, B, E. (2011). El frijol (Phaseolus Vulgaris): Su importancia y como fuente de fotoquímicas. Revista Fuente, Año 3 No. 8 Julio-Septiembre2011 (México).

Valladolid, Ch. R. A. (2016). Leguminosas de grano. Semillas nutritivas para un futuro sostenible. Ministerio de Agricultura y Riego. Lima (Perú). 1 : 1-75.

Yang, YC, HS Lee, SH Lee, JM Clark, y YJ Ahn. (2005). «Ovicidal and adulticidalactivities of Cinnamomum zeylanicum bark essential oil compounds and relatedcompounds against Pediculus humanus capitis (Anoplura: Pediculicidae)». International journal for parasitology 35 (14): 1595-1600.

Yang, YC, SH Lee, WJ Lee, y DH Choi. (2003). Ovicidal and adulticidal effects of Eugenia caryophyllata bud and leaf oil compounds on Pediculus capitis. Journal of agricultural and food chemistry 51 (17): 4884-88. 